\title{
EL IMPUESTO DIFERIDO COMO INDICADOR DE PERSISTENCIA DE RESULTADOS EN LAS EMPRESAS COTIZADAS ESPAÑOLAS
}

\section{DEFERRED TAX AS AN INDICATOR OF RESULTS PERSISTENCE IN SPANISH LISTED COMPANIES}

\author{
Silvina Beatriz Marcolini Tulliani ${ }^{1}$
}

\section{RESUMEN}

El método del impuesto diferido para el reconocimiento contable del impuesto a las ganancias ha sido utilizado por diferentes líneas de investigación. La literatura incluye estudios que lo vinculan con la calidad y características de resultados, la utilización que el mercado hace a partir de la información sobre el mismo o su consideración como indicador de manipulación de ganancias.

El objetivo del trabajo es revisar si las diferencias temporarias entre contabilidad e impuestos, resultan útiles para predecir los resultados futuros en las empresas españolas cotizadas. Se refiere a los períodos 2007-2015.

Se utiliza la ecuación básica que considera como variable dependiente los resultados del período $\mathrm{t}+1$ y como variable independiente, los resultados del período t. Se agrega como variable independiente el impuesto diferido generado en el período. Se trabaja con la totalidad de la muestra y con observaciones-año con resultados positivos exclusivamente. Asimismo se clasifican las diferencias temporarias en positivas y negativas para analizar su incidencia.

Los resultados evidencian que las diferencias temporarias son un indicador importante de persistencia de resultados en las empresas cotizadas españolas con resultados positivos, para los períodos revisados. Los resultados son más determinantes aún, si se consideran sólo las diferencias temporarias positivas.

Palabras claves: Diferencias temporarias, impuesto diferido, persistencia de resultados

Recepción: 11/01/2017. Aprobación: 23/06/2018.

\section{ABSTRACT}

The deferred tax method for income tax accounting recognition has been analized from different perspectives. The literature links it with quality and characteristics of results, the market use of this or the consideration as a manipulation indicator.

The purpose of this paper is to examine if "book-tax differences" are usuful for predicting future results, in Spanish companies for 2007-2015.

The equation considers the results of $t+1$ period as dependent of $t$ period results and added the deferred tax as an independent variable. The sample was used completely and divided between observations with positives and negatives results. The temporary differences were classified too.

The results show that temporary differences are an important indicator of the persistence of results in Spanish listed companies with positive results for the periods reviewed. The results are even more decisive if only the positive temporary differences are considered.

Keywords: Deferred tax, persistence of results, temporary differences.

1 Universidad Nacional de Rosario, Facultad de Ciencias Económicas y Estadística, Rosario, Argentina - smarcoli@fcecon.unr.edu.ar 


\section{INTRODUCCIÓN}

El impuesto sobre la renta o "income tax", grava las rentas obtenidas por las sociedades y las personas físicas. En España, se denomina impuesto sobre beneficios cuando recae sobre personas físicas e impuesto sobre sociedades cuando corresponde a éstas. En la presentación de información financiera para uso externo, se reconoce como resultado y se incluye en el Estado de Resultados o Estado de Resultados Integral.

Su reconocimiento contable a partir del método del impuesto diferido determina la registración de activos por impuestos anticipados o pasivos por impuestos diferidos. Las diferencias entre contabilidad e impuestos aparecen generalmente mencionadas en la literatura como "booktax differences" (BTD) y han sido utilizadas por distintas líneas de investigación en contabilidad. Se ha analizado por ejemplo, si las diferencias entre contabilidad e impuestos tienen alguna asociación con la calidad de los resultados, su crecimiento y persistencia. La literatura previa ha demostrado la vinculación entre el impuesto diferido y la persistencia de resultados, y lo ha hecho utilizando modelos y variables diferentes. En la mayoría de los casos se ha trabajado sobre empresas de Estados Unidos.

El objetivo del trabajo es revisar si las diferencias temporarias entre contabilidad e impuestos, resultan útiles para predecir los resultados futuros en las empresas españolas cotizadas. A partir de la literatura previa, plantea un modelo diferente para el análisis y abarca los períodos 2007-2015. Los resultados evidencian que las diferencias temporarias positivas son un indicador importante de persistencia de resultados.

Se presenta a continuación un análisis del método, algunos aspectos normativos y la revisión de la literatura. El trabajo continúa con la metodología aplicada, los resultados obtenidos y las conclusiones y finaliza con la bibliografía utilizada.

Con respecto al método del impuesto diferido, desde el punto de vista normativo, existe consenso generalizado sobre la consideración del impuesto sobre sociedades, como partida de resultado. Su reconocimiento contable, lleva implícita la necesidad de cuantificación y es en este punto donde surgen dos métodos alternativos, Cuota a pagar y Efecto impositivo.

Desde una perspectiva estrictamente contable, dentro del método del efecto impositivo, si el monto que se reconoce como resultados por impuesto difiere del que se registra como pasivo, se requiere de una tercera cuenta que cierre la igualdad patrimonial. Según Gonzalo Angulo (1987) la naturaleza de dicha cuenta determina la existencia de tres variantes del mismo método.

- Capitalización: Aplica la tasa impositiva vigente al momento en que surgen las diferencias y considera a los impuestos diferidos o anticipados como una partida de ajuste por periodificación. Con esta variante, un cambio en la tasa del impuesto al momento de revertir las diferencias impacta en resultados.

- Deuda: Aplica la tasa impositiva previsible en la fecha esperada de la reversión de la diferencia y revisa el importe pendiente de impuestos diferidos o anticipados ante modificaciones posteriores en la tasa. Estos representan derechos de cobro $\mathrm{u}$ obligaciones de pago futuras de la empresa. Con esta variante, un cambio en la tasa del impuesto al momento de revertir las diferencias, será tratada como un cambio de estimación contable.

- Valor neto de impuestos: Aplica la tasa impositiva vigente al momento en que surgen las diferencias y considera a los impuestos diferidos o anticipados como un componente del valor de los activos y pasivos con los que se relaciona. Estos representan un aumento o una disminución del valor de los activos y pasivos a partir de los cuales se generan las diferencias. Con esta variante, un cambio en la tasa del impuesto al momento de revertir las diferencias impacta en resultados.

Por otra parte, Giner y Mora (1991) atendiendo al cómputo de las diferencias, plantean dos corrientes dentro del mismo método del efecto impositivo:

- Cómputo parcial: Excluye las diferencias sobre las que existe evidencia razonable de no reversión en un período de tiempo.

- Cómputo total: Incluye todas las diferencias independientemente del tiempo de reversión.

Ahora bien, no todas las diferencias que surgen a partir de la aplicación de criterios contables y fiscales deben ser analizadas a partir de los métodos mencionados. Las diferencias pueden ser permanentes o temporales y siguiendo a Comprix et al. (2011), su clasificación depende de que sean o no una cuestión de tiempo.

Las diferencias permanentes son consecuencia de discrepancias conceptuales referidas a qué 
elementos deben ser reconocidos como activos, pasivos, ingresos y gastos, o bien producto de la política fiscal imperante en cuanto al régimen de exenciones o gastos no deducibles. Constituyen el primer ajuste del resultado contable y dejan en evidencia la tasa efectiva del impuesto.

Las diferencias temporales son consecuencia de discrepancias referidas a los criterios de valoración de activos y pasivos, o al momento de reconocimiento de ingresos y gastos. Se trata de una cuestión de periodificación cuya consecuencia inmediata es, el anticipo o diferimiento en el pago del impuesto.

En cuanto a las diferencias temporales, se coincide con García Olmedo (1997) cuando al referirse a la modificación introducida por el Financial Accounting Standard (FAS) 96, rescata el abandono de las "timing differences" y la adopción del concepto "temporary differences" y traduce como "diferencias temporales" a las primeras y "diferencias temporarias" a las segundas.

Más allá de la cuestión semántica, existe una diferencia conceptual importante, ya que las timing differences se refieren a las cuentas de ingresos y gastos, es decir al estado de resultados y las temporary differences a las cuentas de activos y pasivos, es decir al balance. Si bien, en la medida que las diferencias en las valuaciones de activos y pasivos impacten en resultados, la contabilización en cualquiera de los casos determina los mismos saldos en el balance y el mismo gasto por impuesto en resultados, los métodos son muy distintos. Además, de acuerdo a las normas contables vigentes, no todos los ajustes que surgen por la medición periódica de un activo o pasivo impactan directamente en resultados. Conceptualmente se puede decir que siempre que existe una diferencia temporal, habrá también una diferencia temporaria pero sin embargo, no siempre que exista una diferencia temporaria, habrá una diferencia temporal.

Entre las distintas alternativas para reflejar contablemente estas diferencias ha predominado el método de la deuda, dentro del cual es posible distinguir dos enfoques:

- Income statement liability method: Reconoce como activos y pasivos por impuestos, las diferencias temporales que se producen en las cuentas de ingresos y gastos, es decir en el estado de resultados.

- Balance sheet liability method: Reconoce como activos y pasivos por impuestos, las diferencias temporarias que se producen en las cuentas de activos y pasivos, es decir en el balance.
Si todas las variaciones ocurridas en los saldos de activos y pasivos por impuestos diferidos entre un período y otro, tendrían su impacto en el resultado de dicho período, en cualquiera de los casos el gasto por impuesto con cargo a resultados y calculado a partir de la aplicación de los principios contables, debiera ser igual al gasto por impuesto a pagar reconocido en el pasivo y calculado a partir de la aplicación de la normativa fiscal más los incrementos en los pasivos por impuesto diferido y las disminuciones en los activos por impuesto diferido y menos las disminuciones en los pasivos por impuesto diferido y los aumentos en los activos por impuesto diferido.

Considerando los aspectos normativos, en el ámbito internacional, la Norma Internacional de Contabilidad (NIC) $\mathrm{N}^{\mathrm{o}} 12$, prescribe el tratamiento contable del impuesto a las ganancias, que incluye todos los impuestos, nacionales o extranjeros que se relacionen con las ganancias sujetas a imposición. Si se vincula el contenido de la norma con lo expuesto precedentemente sobre impuesto diferido, se está en condiciones de afirmar que la NIC 12, dentro del método del efecto impositivo, utiliza la variante de la deuda, Gonzalo Angulo (1987), con el enfoque de balance y cómputo parcial, Giner y Mora (1991). En este sentido García Olmedo (1997: 669) explica que no se trata de un cómputo parcial de diferencias sino de la comparación con el valor recuperable de los activos.

La norma clasifica a las diferencias temporarias en imponibles y deducibles, según se relacionen con Pasivos o Activos por impuesto diferido respectivamente y reconoce otras causas de generación de Activos por impuesto diferido además de las diferencias temporarias deducibles, como resulta el caso del reconocimiento de activos por impuesto diferido por pérdidas fiscales que puedan compensarse con ganancias fiscales de períodos posteriores.

A su vez, las diferencias temporarias deducibles e imponibles por aplicación de la NIC 12, pueden tener su correlato en:

- Resultado del ejercicio

- Otro resultado integral

- Directamente en el Patrimonio Neto, ajustando el saldo inicial de ganancias acumuladas

- Otra partida de activos y pasivos

Por lo tanto, sólo en el caso de que la contrapartida del impuesto diferido más el impuesto corriente, sea el resultado del ejercicio va a tener efecto sobre la ganancia del ejercicio, mientras 
que sólo en el caso de que la contrapartida del impuesto diferido sea el otro resultado integral lo tendrá sobre el resultado integral. En la medida que el impuesto diferido tenga su contrapartida en el patrimonio neto o en otra partida de activos o pasivos, no tendrá incidencia en los resultados del período ni en el otro resultado integral del período.

Las sociedades españolas que hayan emitido valores cotizables en un mercado regulado de la Unión Europea, deben aplicar las Normas Internacionales de Información Financieras (NIIF) desde el 01 de enero de 2005, para la presentación de sus estados financieros consolidados. Asimismo, la aplicación de las NIIF es voluntaria en los estados financieros consolidados de las empresas no cotizadas y el ejercicio de dicha opción es irreversible. La presentación de los informes financieros individuales y de los consolidados de empresas no cotizadas que no opten por aplicar las NIIF se hará de acuerdo con el Plan General de Contabilidad del año 2007 y sus modificaciones posteriores.

Con respecto a la revisión de la literatura, la búsqueda realizada, ha permitido identificar dos artículos de revisión bibliográfica, Hanlon y Heitzman (2010) y Graham et al. (2012). Ambos clasifican la literatura según se refiera a estudios de asociación o relevancia o gestión de resultados.

Hanlon y Heitzman (2010) clasifican las motivaciones para investigar la contabilidad del impuesto a las ganancias como sigue:

- Investigar si el gasto por impuesto y la divulgación sobre el mismo proveen información sobre la calidad de las ganancias presentes y futuras y en qué medida lo hacen. Incluyen en este punto, las interpretaciones del mercado sobre esta información.

- Investigar si los ajustes impositivos a las ganancias son utilizados para la gestión de las ganancias después de impuestos.

El argumento para la primera línea de investigación es que los ajustes contables son discrecionales mientras que, los ajustes impositivos responden a obligaciones legales por lo que, las diferencias temporarias entre contabilidad e impuestos revelan dicha discrecionalidad.

Un aspecto importante resaltado por los autores es que los estudios utilizan diferentes medidas al referirse a las diferencias entre contabilidad e impuestos, sin explicar la razón de la elección, ni vincularlo con el objetivo de la investigación. Consideran que las investigaciones sobre calidad de resultados, crecimiento y per- sistencia debieran utilizar las diferencias temporarias ya que, podrían ser manipuladas, sin embargo para analizar la utilidad de la información para el mercado, son las diferencias totales las que debieran utilizarse.

Por su parte, Graham et al. (2012), dividen la literatura en tres tópicos:

- Investigar si la contabilidad sobre impuestos es utilizada para manipular resultados, ya sea a través de la utilización de un criterio de valuación o del reconocimiento de contingencias.

- Investigar la asociación de las diferencias entre contabilidad e impuestos y las características del resultado, crecimiento y persistencia.

- Investigar si el mercado utiliza la información sobre impuestos contenida en los informes financieros.

Se advierte que, a diferencia de Hanlon y Heiztman (2010), separa los estudios sobre asociación o relevancia según correspondan a una perspectiva informativa o de la valoración y no se refieren a los cambios en las valuaciones.

El trabajo se ubica dentro de lo que para Hanlon y Heiztman (2010) implica investigar si el gasto por impuesto y la divulgación sobre el mismo proveen información sobre la calidad de las ganancias presentes y futuras y en qué medida lo hacen y para Graham et al. (2012), la asociación entre las diferencias entre contabilidad e impuestos y las características del resultado, crecimiento y persistencia.

Dentro de esta línea, Penman (2001), al referirse al análisis sobre calidad de las ganancias, plantea la relevancia de la información sobre impuestos, la importancia de la cifra de resultado antes de impuesto y del contenido de la nota que acompaña a los informes financieros y la necesidad de separar el impuesto de acuerdo al tipo de resultados que lo ha generado.

Por su parte, Revsine et al. (1999) se refiere a la información contable sobre impuestos y su vinculación con la calidad de las ganancias, haciendo hincapié en las diferencias temporarias, la revisión de la tasa efectiva del impuesto, el efecto contable de un cambio de tasa y la necesidad de utilizar las notas en general y la divulgación sobre impuestos para mejorar el análisis financiero.

A partir de allí, muchos estudios empíricos han relacionado la calidad del resultado con la información sobre impuestos contenida en los estados financieros. Hanlon (2005), analiza el rol 
de las diferencias temporarias como indicador de persistencia de los resultados, ajustes de devengo y flujos de caja y el uso que el mercado hace de éstas. Trabaja con empresas de Estados Unidos para los períodos 1994-2000 y utiliza el modelo de predicción de resultados, según el cual el resultado del período $t+1$ es dependiente del resultado del período $t$, definiendo para su medición, el resultado antes de impuestos.

Para estimar las BTD divide el gasto por impuesto diferido por la tasa del impuesto del país, en el período considerado. Un incremento en el pasivo por impuesto diferido es consistente con una mayor ganancia contable respecto a la fiscal y por el contrario un incremento en el activo por impuesto diferido surge cuando la ganancia contable es inferior a la fiscal.

Divide la muestra en tres submuestras, de acuerdo al tamaño y signo de las diferencias temporarias. La clasificación de la muestra se basa en el ratio $\mathrm{BTD} /$ promedio de activos, en ambos casos del período $t$ y separa las observaciones en aquellas con grandes diferencias positivas, grandes diferencias negativas y pequeñas o resto. Los resultados demuestran que las empresas-año, con grandes diferencias temporarias positivas y negativas están asociadas con menor persistencia de resultados que el resto e indican una asociación entre grandes diferencias temporarias y baja calidad de resultados.

En la misma línea, Lev y Nissim (2004) investigan la capacidad del ratio ganancia fiscal/ ganancia contable, para predecir el crecimiento de las ganancias y los retornos accionarios. La ganancia contable considerada corresponde a la ganancia después de impuestos y antes de los resultados extraordinarios, por lo que la ganancia fiscal determinada, también es después de impuestos, a fin de que resulten comparables.

El estudio distingue entre los períodos pre y post implementación del Statement of Financial Accounting Standard (SFAS) 109 en 1993 y utiliza las diferencias permanentes, temporarias y otros ajustes. Trabaja con empresas de Estados Unidos, con cierre de ejercicio en el mes de diciembre y abarca los períodos 1973-2000. Adicionalmente, utilizan el ratio resultado fiscal/resultado contable como una variable multinomial, para clasificar la muestra en tres submuestras, según su resultado se encuentre en los extremos, cercano a 1 o cercano a 0 , y el resto de las observaciones. Además, lo hacen considerando 6 años para cada submuestra, el año 0 y los 5 posteriores y crean una variable indicadora del crecimiento.

Los resultados indican que el ratio ganancia fiscal/ganancia contable, predice el crecimiento de resultados para los próximos 5 años, tanto en los períodos anteriores a la implementación del SFAS 109 como en los posteriores.

Por su parte, Schmidt (2006), si bien se refiere a persistencia futura de resultados, lo hace a partir de los cambios en la tasa efectiva del impuesto. Entre las razones que utiliza para argumentar el análisis, menciona que las tasas impositivas son de tal magnitud que un pequeño cambio en la tasa efectiva del impuesto puede significar un cambio sustancial de resultados. El análisis incluye a las diferencias permanentes y trata a los impuestos como un componente adicional de resultados.

Explica la ganancia del período $\mathrm{t}+1$ a partir del cambio en la ganancia antes de impuesto del período t utilizando la tasa efectiva del impuesto del período t-1 y del cambio en la tasa efectiva anual entre el período $\mathrm{t}-1 \mathrm{y} \mathrm{t}$, por la ganancia antes de impuesto del período $t$, pero descompuesta en la tasa anual estimada al término del primer trimestre y la tasa anual revisada. Trabaja con empresas de Estados Unidos y abarca los períodos 1994-2001. Excluye las empresas con resultados negativos antes de impuesto y divide la muestra según la tasa efectiva anual revisada aumente o disminuya respecto a la tasa efectiva anual estimada al término del primer trimestre.

Los resultados evidencian una asociación positiva y significativa entre el cambio de tasa efectiva del impuesto y las ganancias futuras. Se demuestra que el cambio de tasa anual estimada en el primer trimestre es más persistente y por lo tanto más útil para predecir resultados futuros, que el cambio de tasa anual revisado.

Si bien la literatura previa ha demostrado la vinculación entre el impuesto diferido y la persistencia de resultados, el trabajo que se presenta incorpora un modelo diferente para el análisis y se refiere a las empresas españolas.

\section{METODOLOGÍA, MATERIAL Y MÉTODOS}

Los datos se obtienen de la base de datos Sabi, el 28 de enero de 2017. A dicha fecha, la base había incorporado la actualización 2091 de fecha 23 de enero de 2017. Con idéntica estrategia de búsqueda se obtuvieron las empresas cotizadas y no cotizadas españolas. Los pasos seguidos para la selección fueron los siguientes:

1. País: España.

2. Clasificación Nacional de Actividades Económicas (CNAE 2009): Todos los códigos 
excepto los correspondientes a actividades financieras y de seguros que aparecen con los números 64, 65 y 66 .

3. Tipo de cuentas: Normal PGC 2007 (ES), Consolidado PGC 2010 (ES).

4. Años con cuentas disponibles: 2007 a 2015.

Considerando que la tributación en España recae sobre la información individual, se trabaja sobre los estados financieros individuales. La base arroja un total de 25.455 empresas, de las cuales 131 son cotizantes y 25.324 son no cotizantes. Al trabajar con las 131 empresas cotizadas y los años de cuentas disponibles se obtiene un total de 1085 observaciones. El armado de la tabla de datos determina la eliminación de 127 observaciones porque no contienen ninguno de los datos solicitados y 14 observaciones porque no contienen el dato del resultado antes de impuestos en al menos dos años consecutivos. Luego de lo expuesto, la primera tabla de datos reúne 944 empresas-años. Resulta importante aclarar que se mantienen 129 empresas de las 131 seleccionadas originalmente. El diseño de la investigación determina la necesidad de trabajar con el resultado del periodo $\mathrm{t}+1$ y por lo tanto la pérdida de los datos del último año relevado. En la mayoría de los casos coincide con el 2015 y genera la eliminación de 129 observaciones.

Finalmente, el cálculo del impuesto diferido de cada ejercicio y surgido a partir de la comparación entre los saldos al inicio y al cierre de los activos y pasivos por impuesto diferido, determina la pérdida de los datos del primer ejercicio relevado que, generalmente coincide con los años 2007 o 2008. A partir de esta última eliminación, de otras 129 observaciones, la muestra queda reducida a 686 empresas-años y es representativa de 122 empresas.

Con el objetivo de alisar las variables, el total del resultado antes de impuestos (RAI) del ejercicio $t+1$, se divide por el total de activos del mismo periodo, y el RAI correspondiente al período $t$, por el total de activos del periodo $t$.

De acuerdo con Hanlon y Heitzman (2010), se trabaja con las diferencias temporarias (DT) del período. Para calcularlas, se divide el impuesto diferido por la alícuota del impuesto vigente en el país para el período considerado. En el año 2014 se produjo un cambio de tasa del impuesto sobre sociedades en España, que pasó del 30 al $28 \%$. Se utiliza la tasa del $28 \%$ a partir del año 2014 y del $30 \%$ para los anteriores. Para que esta variable DT sea comparable a la variable RAI, también se la divide por el total de activos del periodo $t$.
Los datos disponibles, en esta etapa de la investigación solo permiten determinar el monto de la DT generado en el ejercicio por lo que el análisis considera que dicha variación impacta directamente en una de las alternativas aceptadas por la NIC 12, que es el resultado del ejercicio. La DT puede tener sigo positivo, lo que significa que en el período ha aumentado el activo por impuesto diferido o disminuido el pasivo por impuesto diferido y por lo tanto el impuesto a las ganancias determinado es menor al impuesto corriente y el resultado del ejercicio es mayor. Si la DT tiene signo negativo, determina una disminución del resultado del ejercicio.

Se estima la persistencia de los resultados antes de impuestos a partir de la siguiente ecuación:

$$
\operatorname{RAI}_{\mathrm{t}+1}=\mathrm{y}_{0}+\mathrm{y}_{1} \mathrm{RAI}_{\mathrm{t}}+€_{\mathrm{t}+1}
$$

RAI $=$ Resultado antes de impuestos

Se propone agregar en el modelo, como variable independiente las diferencias temporarias a partir de su consideración en la literatura, como indicador de persistencia de resultados (Lev y Nissim, 2004 y Hanlon, 2005).

$$
\mathrm{RAI}_{\mathrm{t}+1}=\mathrm{y}_{0}+\mathrm{y}_{1} \operatorname{RAI}_{\mathrm{t}}+\mathrm{y}_{2} \mathrm{DT}_{\mathrm{t}}+€_{\mathrm{t}+1}
$$

\section{$\mathrm{DT}=$ Diferencia temporaria}

De acuerdo con Hanlon (2005), que clasifica las diferencias temporarias en positivas y negativas, se clasifican dichas diferencias dentro del grupo de empresas-año con resultados positivos, creando una variable dicotómica que asume valor 1 si la DT t es positiva y valor 0 si resulta negativa. La regresión planteada es la siguiente:

$$
\begin{aligned}
& \operatorname{RAI}_{\mathrm{t}+1}=\mathrm{y}_{0}+\mathrm{y}_{1} \operatorname{RAI}_{\mathrm{t}}+\mathrm{y}_{2} \text { DTpos }_{\mathrm{t}} \\
& \quad+\mathrm{y}_{3} \operatorname{RAI}_{\mathrm{t}}{ }^{*} \operatorname{DTpos}_{\mathrm{t}}+€_{\mathrm{t}+1} \text { (3) }
\end{aligned}
$$

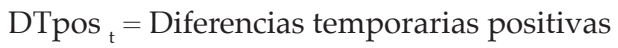

Asimismo Hanlon (2005), clasifica las diferencias dentro de cada grupo en quintiles. Con el objetivo de verificar si el monto de la DT resulta significativo en el análisis de persistencia y considerando el tamaño de las submuestras, se las ha clasificado en cuartiles. Dentro de la submuestra de observaciones con resultados positivos se obtuvieron 98 empresas-año por cuartil. Se presenta a continuación la regresión que incorpora una variable dummy que es igual a 1 para las observaciones que se ubican en el 
cuartil más alto de diferencias temporarias e igual a 0 en los restantes casos, siempre dentro de la muestra de observaciones con resultados positivos. La ecuación es la siguiente:

$$
\begin{gathered}
\operatorname{RAI}_{\mathrm{t}+1}=\mathrm{y}_{0}+\mathrm{y}_{1} \operatorname{RAI}_{\mathrm{t}}+\mathrm{y}_{2} \mathrm{DTg}_{\mathrm{t}}+\mathrm{y}_{3} \mathrm{RAI}_{\mathrm{t}} \\
{ }^{*} \mathrm{DTg}_{\mathrm{t}}+€_{\mathrm{t}+1}(4)
\end{gathered}
$$

$\mathrm{DTg}_{\mathrm{t}}=$ Diferencias temporarias grandes

\section{RESULTADOS}

La variable $\mathrm{RAI}_{\mathrm{t}^{\prime}}$ muestra una observación extrema y otras cinco que, aunque no resultan tan extremas, representan un alejamiento de la media. Se eliminan 6 observaciones y la muestra queda reducida a 680 empresas-año. Se presentan en la Tabla 1, los estadísticos descriptivos:

Tabla 1. Estadísticos descriptivos - Muestra 680 observaciones.

\begin{tabular}{|c|c|c|c|c|c|}
\hline Variable & Observaciones & Mean & Std. Dev. & Min & Max \\
\hline $\mathrm{RAI}_{\mathrm{t}+1}$ & 680 & 0.0179183 & 0,3695262 & $-1,808618$ & 8,224006 \\
\hline $\mathrm{RAI}_{\mathrm{t}}$ & 680 & 0,0083932 & 0,1705118 & $-1,808618$ & 0,964812 \\
\hline $\mathrm{DT}_{\mathrm{t}}$ & 680 & 0,005529 & 0,1245305 & $-2,108717$ & 0,6985739 \\
\hline
\end{tabular}

Fuente: Elaboración propia.

Los resultados de la regresión (1) se presentan en la Tabla 2:

Tabla 2. Resultados regresión (1)

Número de observaciones $=680$ - R-squared $=0.0093$

\begin{tabular}{|c|c|c|c|c|}
\hline RAI $_{t+1}$ & Coef. & Std. Err. & $t$ & $P>(t)$ \\
\hline RAI $_{t}$ & 0.2084856 & 0.0828432 & 2,52 & 0,012 \\
\hline Const & 0,0161685 & 0,0141325 & 1,14 & 0,253 \\
\hline
\end{tabular}

Fuente: Elaboración propia.

Los resultados de la regresión (2) se presentan en la Tabla 3:

Tabla 3. Resultados regresión (2)

Número de observaciones $=680$ - R-squared $=0,0096$

\begin{tabular}{|c|c|c|c|c|}
\hline RAI $_{t+1}$ & Coef. & Std. Err. & $t$ & $P>(t)$ \\
\hline RAI $_{t}$ & 0,2064451 & 0,0829921 & 2,49 & 0,013 \\
\hline DT $_{t}$ & $-0,056197$ & 0,1136358 & $-0,49$ & 0,621 \\
\hline Const & 0,0164963 & 0,141559 & 1,17 & 0,244 \\
\hline
\end{tabular}

Fuente: Elaboración propia. 
Si bien los resultados evidencian que el $\mathrm{RAI}_{t}$ es significativo para predecir los $\mathrm{RAI}_{t+1}$, la variable DT no resulta significativa al respecto. No se muestra una mejora en el coeficiente $\mathrm{RAI}_{t^{\prime}}$ respecto a la regresión anterior.

La variable DT tiene valor cero en algunas de las empresas-año, lo que indica que no existen DT en esos casos. Como se pretende revisar si las DT resultan significativas para la predicción de resultados se ha clasificado la muestra según la variable DT sea igual a 0 o no. De acuerdo a lo expuesto, se han excluido aquellas observaciones cuya variable DT $=0$. La muestra queda compuesta por 614 empresas-año. Se presentan los descriptivos de las variables con la nueva muestra en la Tabla 4 y se repiten las regresiones (1) y (2) en las Tablas 5 y 4 :

Tabla 4. Estadísticos descriptivos - Muestra 614 observaciones.

\begin{tabular}{|c|c|c|c|c|c|}
\hline Variable & Observaciones & Mean & Std. Dev. & Min & Max \\
\hline RAI $_{t+1}$ & 614 & 0,0293761 & 0,3725774 & $-1,752969$ & 8,224006 \\
\hline RAI $_{t}$ & 614 & 0,0154491 & 0,1624466 & $-1,808618$ & 0,964812 \\
\hline $\mathrm{DT}_{\mathrm{t}}$ & 614 & 0,0061233 & 0,1310492 & $-2,108717$ & 0,6985739 \\
\hline
\end{tabular}

Fuente: Elaboración propia.

Tabla 5. Resultados regresión (1)

Número de observaciones $=614$ - R-squared $=0,0075$

\begin{tabular}{|c|c|c|c|c|}
\hline RAI $_{t+1}$ & Coef. & Std. Err. & $t$ & $P>(t)$ \\
\hline RAI $_{t}$ & 0.1988273 & 0,0923617 & 2,15 & 0,032 \\
\hline Const & 0,0263044 & 0,0150594 & 1,75 & 0,081 \\
\hline
\end{tabular}

Fuente: Elaboración propia.

Tabla 6. Resultados regresión (2)

Número de observaciones $=614$ - R-squared $=0,0080$

\begin{tabular}{|c|c|c|c|c|}
\hline RAI $_{t+1}$ & Coef. & Std. Err. & $t$ & $P>(t)$ \\
\hline RAI $_{t}$ & 0,1960465 & 0,0925666 & 2,12 & 0,035 \\
\hline DT $_{t}$ & $-0,0605149$ & 0,1147441 & $-0,53$ & 0,598 \\
\hline Const & 0,0267179 & 0,0150887 & 1,77 & 0,0077 \\
\hline
\end{tabular}

Fuente: Elaboración propia.

La eliminación de las observaciones con DT igual a cero, no mejora los resultados obtenidos respecto a las regresiones anteriores.

De acuerdo con Hanlon (2005) y Schmidt (2006), se eliminan de la muestra las observaciones con resultados negativos en el periodo t. Esta eliminación es consistente con los análisis sobre persistencia de resultados ya que, los resultados negativos no pueden ser persistentes porque implicarían la quiebra de la empresa. Esta clasificación determina dos submuestras de 391 observaciones con resultados positivos en el periodo $t \mathrm{y}$ 223 observaciones con resultados negativos.

Las Tablas 7 y 8 muestran los resultados de las regresiones (1) y (2) para las empresas-año con resultados positivos: 
Tabla 7. Resultados regresión (1)

Número de observaciones $=391$ - R-squared $=0.3113$

\begin{tabular}{|c|c|c|c|c|}
\hline RAI $_{t+1}$ & Coef. & Std. Err. & $t$ & $P>(t)$ \\
\hline RAI $_{t}$ & 0,6920012 & 0,0521823 & 13,26 & 0,000 \\
\hline Const & 0,0047652 & 0,0071551 & 0,67 & 0,506 \\
\hline
\end{tabular}

Fuente: Elaboración propia.

Tabla 8. Resultados regresión (2)

Número de observaciones $=391$ - R-squared $=0.3172$

\begin{tabular}{|c|c|c|c|c|}
\hline RAI $_{\mathrm{t}+1}$ & Coef. & Std. Err. & $\mathrm{t}$ & $\mathrm{P}>(\mathrm{t})$ \\
\hline $\mathrm{RAI}_{\mathrm{t}}$ & 0,6968216 & 0,0520958 & 13,38 & 0,000 \\
\hline $\mathrm{DT}_{\mathrm{t}}$ & 0,1549795 & 0,0852423 & 1,82 & 0,070 \\
\hline Const & 0,0046996 & 0,0071341 & 0,66 & 0,510 \\
\hline
\end{tabular}

Fuente: Elaboración propia.

La eliminación de las empresas-año con resultado negativo, determina una modificación sustancial en los resultados y evidencia una alta persistencia de resultados y una incidencia mo- derada de la DT.

La Tabla 9 incluye los resultados de la regresión (3) para las empresas-año con ganancias:

Tabla 9. Resultados regresión (3)

Número de observaciones $=391$ - R-squared $=0.3560$

\begin{tabular}{|c|c|c|c|c|}
\hline RAI $_{t+1}$ & Coef. & Std. Err. & $t$ & $P>(t)$ \\
\hline RAI $_{t}$ & 0,389448 & 0,0779234 & 5,00 & 0,000 \\
\hline DTpos $_{t}$ & $-0,0327623$ & 0,0139106 & $-2,36$ & 0,019 \\
\hline RAI $_{t}{ }^{*}$ DTpos & 0,5190294 & 0,1025579 & 5,06 & 0,000 \\
\hline Cons & 0,023514 & 0,0098532 & 2,39 & 0,017 \\
\hline
\end{tabular}

Fuente: Elaboración propia.

Los resultados obtenidos a partir de la eliminación de las DT negativas demuestran un alto coeficiente de persistencia de resultados, siendo las DT positivas las que más lo explican. Recordemos que estas diferencias indican un impacto positivo en el resultado del ejercicio.

Para revisar si los resultados son sensibles al cambio en las variables, cuando se consideran las DT del cuartil más alto se presentan en la Tabla 10, los resultados de la regresión (4): 
Tabla 10. Resultados regresión (4)

Número de observaciones $=391$ - R-squared $=0,3297$

\begin{tabular}{|c|c|c|c|c|}
\hline $\mathrm{RAI}_{\mathrm{t}+1}$ & Coef. & Std. Err. & $\mathrm{t}$ & $\mathrm{P}>(\mathrm{t})$ \\
\hline $\mathrm{RAI}_{\mathrm{t}}$ & 0,5585366 & 0,0663079 & 8,42 & 0,000 \\
\hline $\mathrm{DTg}_{t}$ & $-0,0361988$ & 0,0157474 & $-2,30$ & 0,022 \\
\hline $\mathrm{RAI}_{\mathrm{t}}{ }^{*} \mathrm{DTg}{ }_{\mathrm{t}}$ & 0,340376 & 0,1056698 & 3,22 & 0,001 \\
\hline Cons & 0,0176551 & 0,0084915 & 2,08 & 0,038 \\
\hline
\end{tabular}

Fuente: Elaboración propia.

La consideración de las diferencias temporarias más altas en la ecuación, evidencia similares resultados. En las empresas-año con resultados positivos resultan significativas las diferencias temporarias positivas en la predicción de resultados. Sin embargo, los resultados determinan que cuando las DT son las más altas, la persistencia del resultado disminuye o, en otras palabras, los resultados se vuelven menos persistentes. Asimismo el R cuadrado disminuye. Estos resultados son consistentes con los obtenidos por Hanlon (2005).

Además, para revisar la robustez del modelo y a partir de la separación en cuartiles ya comentada, realizada considerando la variable DT, se crean dos variables dummy. La primera, asume valor 1 para el cuartil con valores positivos más altos y valor 0 para el resto. La segunda, asume valor 1 para los valores negativos más bajos y valor 0 para el resto. Los resultados siguen evidenciando una muy alta vinculación entre las DT positivas y la persistencia de resultados. El coeficiente es negativo cuando las DT son negativas, siendo estas últimas no significativas en el análisis de persistencia.

\section{DISCUSION, CONCLUSIÓN}

Las DT son un indicador importante de persistencia de resultados en las empresas cotizadas españolas con resultados positivos, para los períodos 2007-2015. Los resultados son más determinantes aún, si se consideran sólo las DT positivas. Una diferencia temporaria positiva en el periodo $t$ indica un menor impuesto devengado o un mayor resultado, en este caso positivo. Esto implica que incide en el mantenimiento o incremento de resultados positivos o en otras palabras contribuye a que los resultados positivos sean más persistentes. La clasificación de la muestra a partir de las DT que caen en el cuartil más alto evidencia similares resultados. En las empresas-año con resultados positivos resultan significativas las diferencias temporarias positivas en la predicción de resultados. Sin embargo, los resultados determinan que cuando las DT son las más altas, la persistencia del resultado disminuye o, en otras palabras, los resultados se vuelven menos persistentes. Asimismo el R cuadrado disminuye.

La consideración de las diferencias temporarias positivas más altas y negativas más bajas en una misma ecuación, de acuerdo al planteo de Hanlon (2005), no modifica los resultados. Una diferencia temporaria negativa en el periodo $t$ indica un mayor impuesto devengado o un menor resultado. Esto implica que no incide en el mantenimiento o incremento de resultados positivos o en otras palabras contribuye a que los resultados positivos sean menos persistentes.

El análisis realizado en este trabajo se replicará sobre las empresas no cotizadas españolas, con el objetivo de verificar si los resultados se repiten en estados financieros preparados bajo normas locales y tratándose de entes más pequeños.

Resulta interesante para futuras investigaciones revisar si los resultados se modifican al considerar para el análisis, exclusivamente la porción de las diferencias temporarias imputadas al resultado del ejercicio. Para ello es necesario consultar la información a revelar correspondiente a impuesto diferido. Los resultados debieran mejorar ante esta variante, evidenciando la importancia de la revelación sobre impuesto diferido, para los analistas de información financiera. 


\section{REFERENCIAS BIBLIOGRAFICAS}

BALL, R., BROWN, P. (1968). An Empirical Evaluation of Accounting Income Numbers, Journal of Accounting Research, 6, 2, 159-178.

BEAVER, W. (1968). The Information Content of Annual Earnings Announcements, Journal of Accounting Research, 6, 67-92.

BENTABOL MANZANARES, M. A., CALLE GARCIA, M. J., LUNA JIMENEZ, M. J. (1991). El impuesto de sociedades en el nuevo plan general de contabilidad, Cuadernos, 21, 29-49.

BROWN, P. (1989). Invited Remarks, Journal of Accounting Research", 27, 202-217.

COMPRIX, J., GRAHAM, R., MOORE, J. (2011). Empirical Evidence on the Impact of Book-Tax Differences on Divergence of Opinion among Investors, American Accounting Association, JATA, $33,1,51-78$.

FINANCIAL ACCOUNTING STANDARDS BOARD:

FAS 96 (publicado en diciembre de 1987), FAS 109 (publicado en febrero de 1992)

GARCIA-OLMEDO, R. (1997). Las diferencias temporarias: Otro enfoque en el tratamiento contable del impuesto sobre beneficios, Técnica Contable, XLIX, 586, 665-680.

GINER INCHAUSTI, B., MORA EGUINDAÑOS, A. (1991). La contabilización del impuesto sobre beneficios: el marco teórico, Técnica Contable, XLIII, 314, 547-562.

GONZALO ANGULO, J. A. (1987). Algunas cuestiones relativas a la contabilización del efecto impositivo, en Ponencias y Comunicaciones al IV Congreso de AECA, Barcelona, 307-333.

GRAHAM, J., RAEDY, J., SHACKELFORD, D. (2012). Research in accounting for income taxes, Journal of Accounting and Economics, 53, 412-434.

HANLON, M. (2005). The Persistence and Pricing of Earnings, Accruals and Cash Flows When Firms Have Large Book-Tax Differences, The Accounting Review, 80, 1, 137-166.
HANLON, M., HEITZMAN, S. (2010). A review of tax research, Journal of Accounting and Economics, 50, 127-178.

INTERNATIONAL ACCOUNTING STANDARDS BOARD: Norma Internacional de Contabilidad $N^{\circ}$ 12: Impuesto a las Ganancias

LEV, B. (1989). On the Usefulness of Earnings and Earnings Reseach: Lessons and Directions from Two Decades of Empirical Research, Journal of Accounting Research, 27, 153-192.

LEV, B., NISSIM, D. (2004). Taxable Income, Future Earnings, and Equity Values, The Accounting Review, 79, 4, 1039-1074.

PENMAN, S. (2001). Financial Statement Analysis and Security Valuation, McGraw-Hill, Irwin.

REVSINE, L., COLLINS, D., JOHNSON, W. (1999). Financial Reporting \& Analysis, Prentice Hall.

SCHMIDT, A. (2006). The persistence, forecasting and valuation implication of the tax change components of earnings. The Accounting Review. $81,589-616$.

ZAMORA RAMIREZ, C., SIERRA MOLINA, G. (2000). Una perspectiva crítica desde el marco conceptual respecto a la contabilización del impuesto sobre beneficios, Revista de Contabilidad, 3, 5, 183-209. 